\title{
SOME SIMPLE APPLICATIONS OF ELEMENTARY ALGEBRA TO ARITHMETIC.
}

\author{
By M. O. TRIPP, \\ Olivet College, Olivet, Mich.
}

The object of this article is to show how we may make our first term's work in algebra more concrete and interesting, and at the same time vitally connected with the work in arithmetic which the student has had in the grades. The teacher of beginning algebra always experiences considerable difficulty in familiarizing students with generalized numbers. In passing from the ordinary arithmetic of the grades to the literal arithmetic of first term algebra students frequently fail to make connection, and hence fall into the habit of mechanical manipulation. Arithmetic becomes far more interesting when the student, by means of his algebra, can prove some of the statements which he has merely taken for granted in the elementary school.

One way to make division in algebra more vital is to connect it with the tests for the divisibility of numbers.

Let us consider the test: Any integer is divisible by 2 if its right-hand digit is $0,2,4,6,8$. If the number has two digits it can be represented as

and hence division by 2 gives

$$
10 x+y
$$

$$
\frac{10 x+y}{2}=5 x+\frac{y}{2}
$$

The pupil will have no difficulty in realizing that if the quotient on the right is to be an integer $y$, or the right-hand digit, must be $0,2,4,6,8$. After the student has proved the rule for a number of two digits he can take up a number with three digits in the same way, and finally an integer consisting of any number of digits.

To test a number of three digits for divisibility by 3 we assume the number in the form

$$
100 x+10 y+z
$$

Division by 3 may be indicated as follows:

$$
\frac{100 x+10 y+z}{3}=33 x+3 y+\frac{x+y+z}{3} .
$$

In order that the right side may be an integer it is necessary that

$$
\frac{x+y+z}{3}
$$

shall be an integer; that is, that the sum of the digits of the 
given number shall be divisible by 3 . It is evident that, in like manner, the same test can be shown to hold no matter how many digits the number has.

The tests for divisibility by 4 and 5 are very simple. The practical test for 6 is not obtained by ordinary division, but can most readily be obtained from the tests for 2 and 3 .

If we take three digits we get a test for divisibility by 7 from the following division:

$$
\frac{100 x+10 y+z}{7}=14 x+y+\frac{2 x+3 y+z}{7},
$$

That is, a number of three digits is divisible by 7 if the sum of twice the hundreds' digit, three times the tens' digits, and the units' digit is divisible by \%. A working rule can already be obtained, by division as above, for an integer having any number of digits.

Let us test a number of five digits for divisibility by 11 . Division of the number in algebraic form by 11 gives

$$
\begin{aligned}
\frac{10,000 x+1,000 y+100 z+10 w+u}{11} \\
=909 x+91 y+9 z+w+\frac{x-y+z-w+u}{11}
\end{aligned}
$$

Hence a number of five digits is divisible by 11 if the sum of the odd numbered digits, beginning at the left, minus the sum of the even numbered digits is divisible by 11 .

It is evident that tests for divisibility by other numbers can be readily obtained. Division in algebra taught with such illustrations as the above tends to make the student feel at the outset that the subject he is studying is of some use.

The consideration of Pythagorean numbers, that is, triples of rational numbers which may represent the three sides of a right triangle, leads to some interesting applications of the special products found under multiplication in the first term's work in algebra. In preparing problems in arithmetic on the right triangle it is convenient to know how to pick out triples of Pythagorean numbers belonging to dissimilar triangles. The identity $\left(a^{2}-b^{2}\right)^{2}+(2 a b)^{2}=\left(a^{2}+b^{2}\right)^{2}$

can be used for the selection of such triples. If we give $a$ and $b$ any values different from each other the three sides of a right triangle may be represented by the numbers

$$
\left(a^{2}-b^{2}\right), 2 a b,\left(a^{2}+b^{2}\right) \text {. }
$$

Thus let $a=2$ and $b=1$, and we get the well known triple 3,4 , 5. If $a=4, b=1$, we get the triple $15,8,1 \%$. 
By putting together Pythagorean right triangles having a common side we may find triples of Heronian ${ }^{1}$ numbers, that is, rational numbers representing the sides of a plane triangle such that the area shall be rational. From the above Pythagorean triple $3,4,5$ we see that $9,12,15$ may also represent the sides of a right triangle. But if we take the even number 12 and set it equal to $2 a b$ we have $a b=6$, which is satisfied by $a=3, b=2$. Hence $a^{2}-b^{2}=5$ and $a^{2}+b^{2}=13$. Therefore by putting the two right triangles with sides $9,12,15$ and $5,12,13$ together with the side 12 in common, we have a triangle with the sides 13,14 , 15 whose area is rational, that is, $13,14,15$ is a triple of Heronian numbers.
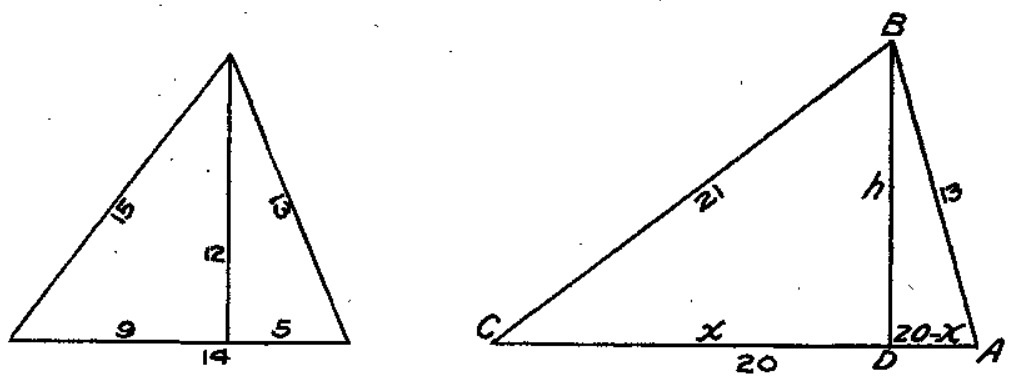

By taking the Pythagorean triangle whose sides are $20,16,12$ and placing it next to the right triangle whose sides are 13, 5, 12 so that the side 12 shall be common we have the Heronian triple $21,13,20$.

An interesting application of the rule for finding the product of the sum and difference of two numbers may be made in finding the squares of small numbers. From the identity

$$
a^{2}=(a+b)(a-b)+b^{2}
$$

we may get the rule: The square of a number equals the sum of the number and any second number, multiplied by their difference, plus the square of the second number. Thus

$$
22^{2}=(22+2)(22-2)+4 \text {. }
$$

This rule enables the student to square numbers up to 100 mentally. The rule works most conveniently on numbers ending in 5 .

Another set of interesting exercises which may be proven very readily with a little algebra is that relating to the difference between any number and the number obtained by reversing the order of the digits. The student can readily discover for him-

\footnotetext{
1 For a list of Pythagorean and Heronian triples see Halsted's Mensuration;
Ginn \& Co.
} 
self when this difference is divisible by 9 and when it is divisible by 9 times 11 .

There are certain problems in fractions which illustrate very nicely how algebra may become a powerful means for the proving of general statements. Let us take the problem: How is the value of a proper fraction affecterl by adding the same positive number to both terms?

Let $x=$ numerator and $y=$ denominator.

Then

$$
\frac{x}{y}=1-k, \quad(0<k<1) .
$$

Therefore

Adding $a$ to each side,

$$
x=y-k y \text {. }
$$

$$
x+a=y+a-k y .
$$

Dividing each side by $y+a$,

It is evident that

$$
\frac{x+a}{y+a}=1-k\left(\frac{y}{y+a}\right) .
$$

$$
1-k\left(\frac{y}{y+a}\right)>1-k .
$$

Hence

$$
\frac{x+a}{y+a}>\frac{x}{y} \text {. }
$$

That is, a proper fraction is increased when the same quantity is added to both numerator and denominator. Other problems can be made by considering improper fractions, or by considering the effect of subtracting the same quantity from each term of the fraction.

Algebra may be used in an interesting way to reduce a repeating decimal to a common fraction.

$$
\begin{array}{rlrl}
\text { Let } & x & =.333333 \\
\text { Then } & 10 x & =3.333333 \\
\text { Subtracting } & 9 x & =3, \\
\text { or } & x & =\frac{1}{3} . \\
\text { Again let } & x & =2.3454545 \\
\text { Then } & 100 x & =234.5454545 \\
\text { Subtracting } & 99 x & =232.2, \\
& \text { or } & x & =\frac{232.2}{99}=2^{342} / 990 .
\end{array}
$$

This method of changing repeating decimals to common fractions is so simple that it can be taught during the first week's work in algebra.

A simple application of algebra to mensuration is that of 
finding the area of a triangle when the three sides are given. Let us take the triangle whose sides are $13,20,21$.

Let $x=\mathrm{CD}$.

Then $20-x=\mathrm{DA}$.

From the right triangles $\mathrm{CDB}$ and $\mathrm{ABD}$ we have

or

$$
21-x^{2}=h^{2}=13^{2}-(20-x)^{2}
$$

Hence $441-x^{2}=169-400+40 x-x^{2}$.

Therefore or

$$
\begin{aligned}
& x=164 / 5 . \\
& h=21^{2}-7056 / 25=3909 / 25, \\
& h=63 \% .
\end{aligned}
$$

Hence the area $=1 / 2 \cdot 63 / 5 \cdot 20=126$.

If we take $a, b, c$ as the sides of the triangle we get the usual Heronian formula,

$$
\text { area }=\sqrt{s(s-a)(s-b)(s-c)} \text { where } s=\frac{a+b+c}{2} .
$$

One reason why beginning algebra often becomes uninteresting is that the teacher does not offer the student enough concrete illustrative material. It is hoped that the above simple applications will be of use to the teacher and will incite him to think up more problems of a simple nature.

\section{MATHEMATICAL EQUIPMENT AND ITS USES.}

BY H. C. WRIGHT, University of Chicago High School.

In January, 1915, it was the privilege and the pleasure of the writer to visit one of the large high schools in the vicinity of Chicago. The high-school district has an equalized assessed valuation of approximately $\$ 8,000,000$ and there is levied upwards of $\$ 190,000$ for the annual support of the high school. The equipment provided for the athletics, for science, for manual training, for English, for the classics, for music, for drawing, for domestic science, for commercial instruction and for reference work seemed abundant and of good quality. But in the rooms of the seven mathematics teachers I found much the same equipment as was common in high schools a quarter of a century ago. Chalk, erasers, string, rulers, a portion of squared blackboard, several wooden protractors, and some blackboard compasses were in some of the rooms but not in all. The equipment in the possession of the pupils, aside from textbooks and pencils appeared to consist of rulers and loose paper. Although the school may have possessed equipment not in evidence at the time of my 